\title{
A Hybrid Diagnosis Approach on Transformer's Insulating Oil
}

\author{
Ming-Jong Lin \\ Department of Electronic Engineering, Southern Taiwan University of Science and Technology, Tainan City, \\ Taiwan \\ Email: 1430107@yahoo.com.tw, u018794@taipower.com.tw
}

Received 12 March 2015; accepted 7 April 2015; published 8 April 2015

Copyright (C) 2015 by author and Scientific Research Publishing Inc.

This work is licensed under the Creative Commons Attribution International License (CC BY).

http://creativecommons.org/licenses/by/4.0/

(c) (i) Open Access

\section{Abstract}

The immersed-oil power transformer is so vital equipment in power system that maintenanceengineers take more monitor from transformer's insulating oil to diagnose what is condition of operation. Then the dissolved gas analysis (DGA) is known for an effective technique on detecting incipient faults in oil-immersed power transformers. In this paper, a practical method is presented which consists of the Roger \& Dernenber Ratio Methods, the Linear SVM diagnosis, the Key Gas method and the Specification ANSI/IEEE C57.104 Standard. Thus, incipient faults in power immersed-oil transformers can be directly identified by a report's form which is so easy understood that we can accurate of diagnosis transformer. The user only keys in the measured data of main gases such as $\mathrm{H}_{2}, \mathrm{CH}_{4}, \mathrm{C}_{2} \mathrm{H}_{2}, \mathrm{C}_{2} \mathrm{H}_{4}, \mathrm{C}_{2} \mathrm{H}_{6}$, and $\mathrm{CO}$ those gases were must decompose via ASTMD3612. The diagnosis result was showed in texts. This paper was taken some data from Taiwan and Siemens Power Company to verify the program that was validation and accuracy of the transformer's insulating oil diagnosis tool.

\section{Keywords}

Power Transformer Diagnosis, Dissolved Gas Analysis, Total Combustible Gases, ANSI/IEEE C57.104 Standard

\section{Introduction}

Immersed-oil power transformer's insulating oil, to strengthen insulation and cut temperature of operation down of transformer for ability of supply. If the Dissolved Gas Analysis (DGA) of insulating oil is not to meet maintenance code that it will pose transformer great threaten. Thus the diagnosis of insulating oil is regarded as an important task. The insulating oil has to through chromatography instrument (ASTM D3612) yield some gases 
such as Ethane $\left(\mathrm{C}_{2} \mathrm{H}_{6}\right)$, Hydrogen $\left(\mathrm{H}_{2}\right)$, Methane $\left(\mathrm{CH}_{4}\right)$, Carbon Dioxide $\left(\mathrm{CO}_{2}\right)$, Ethylene $\left(\mathrm{C}_{2} \mathrm{H}_{4}\right)$, Acetylene $\left(\mathrm{C}_{2} \mathrm{H}_{2}\right)$, Carbon Monoxide (CO), Nitrogen $\left(\mathrm{N}_{2}\right)$, and Oxygen $\left(\mathrm{O}_{2}\right)$ and so on. Then those gases Hydrogen $\left(\mathrm{H}_{2}\right)$, Methane $\left(\mathrm{CH}_{4}\right)$, Ethane $\left(\mathrm{C}_{2} \mathrm{H}_{6}\right)$, Ethylene $\left(\mathrm{C}_{2} \mathrm{H}_{4}\right)$, Acetylene $\left(\mathrm{C}_{2} \mathrm{H}_{2}\right)$, and Carbon Monoxide (CO) are named Total Combustible Gas (TCG) [1]. The dissolved gas analysis (DGA) is a common effective technique on detecting incipient faults in oil-immersed power transformers. The present work investigated the DGA methods and employed the ANSI/IEEE C57.104 Standard Rule, the Linear SVM Method, the Roger \& Doernenber Ratios, and the Key Gas Method to develop a fast immersed-oil power transformer diagnosis approach which was named "a hybrid diagnosis approach". The major character of the program is simultaneously shown what the four types diagnosed results on a report's form by texts. The Hybrid diagnosis approach was validated and confirmed so easy and analysis to use at any condition of transformer insulating oil. Then the approach is proved from a lot of causes which is efficiency and accuracy.

\section{Transformer Fault Diagnosis}

Total Combustible Gases made up of $\mathrm{H}_{2}, \mathrm{CH}_{2}, \mathrm{C}_{2} \mathrm{H}_{6}, \mathrm{C}_{2} \mathrm{H}_{4}, \mathrm{C}_{2} \mathrm{H}_{2}$, CO, its definition as follows, as shown in Formula (1) (unit ppm):

$$
\text { TCG }=\frac{\mathrm{H}_{2}+\mathrm{CH}_{2}+\mathrm{C}_{2} \mathrm{H}_{6}+\mathrm{C}_{2} \mathrm{H}_{4}+\mathrm{C}_{2} \mathrm{H}_{2}+\mathrm{CO}}{10^{6}}
$$

When the immersed oil transformer's insulating oil is along with the transformer operating time and the measured of the cyclical time has a vital relation with its life-span. If the diagnosis of time and approach were unapt that will pose a great threat with transformer.

\subsection{The ANSI/IEEE C57.104 Standard Rule}

When it comes diagnosis of transformer's insulating oil. Every electrical engineer is required to take the ANSI/ IEEE C57.104 Standard Rule as base to compare with analysis because ANSI/IEEE C57.104 Standard Rule is strictly standard and the best effective diagnosis tool for Immersed-oil power transformer's insulating oil.

The ANSI/IEEE C57.104 Standard Rule has shown $\mathrm{H}_{2}, \mathrm{CH}_{4}, \mathrm{C}_{2} \mathrm{H}_{6}, \mathrm{C}_{2} \mathrm{H}_{4}, \mathrm{C}_{2} \mathrm{H}_{2}$, and CO, then any gas of content is from 0 to $\infty$ disguised to four kinds symptom- "Normal", "Attention", "Abnormal", and "Danger", taking $\mathrm{H}_{2}$ for a sample, its content value is less property Normal, over 101 property attention, over 701 property Abnormal, or over 1801 property Danger. The Four kinds of symptom were shown in Table 1. It is widely applicable in diagnosis of transformer insulating oil around the world.

Table 1. Gas content in oil diagnostic [2], unit: ppm.

\begin{tabular}{|c|c|c|c|c|c|}
\hline Name & Content value & Property & Name & Content value & Property \\
\hline \multirow{4}{*}{$\mathrm{H}_{2}$} & $>1801$ & Danger & \multirow{4}{*}{$\mathrm{CH}_{4}$} & $>1001$ & Danger \\
\hline & $>701$ & Abnormal & & $>401$ & Abnormal \\
\hline & $>101$ & Attention & & $>121$ & Attention \\
\hline & $<100$ & Normal & & $<120$ & Normal \\
\hline \multirow{4}{*}{$\mathrm{C}_{2} \mathrm{H}_{6}$} & $>151$ & Danger & \multirow{4}{*}{$\mathrm{C}_{2} \mathrm{H}_{4}$} & $>201$ & Danger \\
\hline & $>101$ & Abnormal & & $>101$ & Abnormal \\
\hline & $>66$ & Attention & & $>51$ & Attention \\
\hline & $<65$ & Normal & & $<50$ & Normal \\
\hline \multirow{4}{*}{$\mathrm{C}_{2} \mathrm{H}_{2}$} & $>35$ & Danger & \multirow{4}{*}{$\mathrm{CO}$} & $>1400$ & Danger \\
\hline & $>10$ & Abnormal & & $>571$ & Abnormal \\
\hline & $>2$ & Attention & & $>351$ & Attention \\
\hline & $<1$ & Normal & & $<350$ & Normal \\
\hline
\end{tabular}




\subsection{The Roger and Dornenburg Ratio Method}

The Roger's ratio method and Dornenburg's ratio method are recognized as an effective tool for diagnosis of transformer's insulating oil. Ones methods are accord with the Standards IEC 60599 and IEEE C57.104 as the framework of the diagnosis and transformer maintenance system. They take the gas ratios such as $\mathrm{CH}_{4} / \mathrm{H}_{2}$, $\mathrm{C}_{2} \mathrm{H}_{2} / \mathrm{C}_{2} \mathrm{H}_{4}, \mathrm{C}_{2} \mathrm{H}_{2} / \mathrm{CH}_{4}, \mathrm{C}_{2} \mathrm{H}_{6} / \mathrm{C}_{2} \mathrm{H}_{2}$ and $\mathrm{C}_{2} \mathrm{H}_{4} / \mathrm{C}_{2} \mathrm{H}_{6}$ for base to compare diagnosis. Each set the value of percentage is shown in Table 2 [3] [4]. The diagnosis principles of the Roger and Dornenburg ratio methods list in Table 2.

\subsection{The Linear SVM Diagnosis}

As of 1999, Japanese electric Association collected 1033 transformers fault of record to analysis what was up from transformer's insulating oil diagnosis. Then it is developed to obtain an insulating oil diagnostic method by Support Vector Machine theory which was called "The Linear SVM (Support Vector Machine) diagnostic method", the method will take $\mathrm{H}_{2}, \mathrm{CH}_{4}, \mathrm{C}_{2} \mathrm{H}_{6}, \mathrm{C}_{2} \mathrm{H}_{4}, \mathrm{C}_{2} \mathrm{H}_{2}$, and $\mathrm{CO}$ of the component data that involve into the formula with the value of the coefficient $(\mathrm{Z})$ to calculated and diagnose inside the condition of transformer, the coefficients of the formula shown in Table 3 [2]. The result of $Z$ is "positive", which means transformer operation normal; on the contrary, $\mathrm{Z}$ is "negative", which may operate in abnormalities of condition. The abnormality’s phenomenon was classified "Overheating”, "Electric Arc”, "Overheating + Discharge” and "Oil Mixes In" four kinds of fault type that it is rely on the " $\mathrm{Z}$ of condition". If the formula shown "negative value" over two, you should get the larger value to make diagnostic analysis. Formula equation is shown behind.

Formula: $\mathrm{Z}=a * \mathrm{H}_{2}+b * \mathrm{CH}_{4}+c * \mathrm{C}_{2} \mathrm{H}_{6}+d * \mathrm{C}_{2} \mathrm{H}_{4}+e * \mathrm{C}_{2} \mathrm{H}_{2}+f * \mathrm{CO}+g$.

Table 2. The roger \& dornenburg ratios [3] [4].

\begin{tabular}{|c|c|c|c|c|c|c|}
\hline & & \multicolumn{5}{|c|}{ Fault Type } \\
\hline & & $\mathrm{T} 1$ & $\mathrm{~T} 2$ & T3 & D1 & D2 \\
\hline Ratio Symbol & Gas Ratio & $\begin{array}{c}\text { Thermal Fault } \\
<300^{\circ} \mathrm{C}\end{array}$ & $\begin{array}{l}\text { Thermal Fault } \\
300^{\circ} \mathrm{C}-700^{\circ} \mathrm{C}\end{array}$ & $\begin{array}{c}\text { Thermal Fault } \\
>700^{\circ} \mathrm{C}\end{array}$ & $\begin{array}{l}\text { Low Energy } \\
\text { Discharge }\end{array}$ & $\begin{array}{c}\text { High Energy } \\
\text { Discharge }\end{array}$ \\
\hline \multicolumn{7}{|c|}{ Dornenburg Ratios } \\
\hline $\mathrm{R} 1$ & $\mathrm{CH}_{4} / \mathrm{H}_{2}$ & & $1.0<\mathrm{R} 1$ & & $\mathrm{R} 1<0.1$ & $0.1<\mathrm{R} 1<1.0$ \\
\hline $\mathrm{R} 2$ & $\mathrm{C}_{2} \mathrm{H}_{2} / \mathrm{C}_{2} \mathrm{H}_{4}$ & & $\mathrm{R} 2<0.75$ & & & $0.75<\mathrm{R} 2$ \\
\hline R3 & $\mathrm{C}_{2} \mathrm{H}_{2} / \mathrm{CH}_{4}$ & & $\mathrm{R} 3<0.3$ & & $\mathrm{R} 3<0.3$ & $0.3<\mathrm{R} 3$ \\
\hline $\mathrm{R} 4$ & $\mathrm{C}_{2} \mathrm{H}_{6} / \mathrm{C}_{2} \mathrm{H}_{2}$ & & $\mathrm{R} 4<0.4$ & & $0.4<\mathrm{R} 4$ & $\mathrm{R} 4<0.4$ \\
\hline \multicolumn{7}{|c|}{ Roger Ratios } \\
\hline $\mathrm{R} 1$ & $\mathrm{CH}_{4} / \mathrm{H}_{2}$ & $1.0<\mathrm{R} 1$ & $1.0<\mathrm{R} 1$ & $1.0<\mathrm{R} 1$ & $\mathrm{R} 1<0.1$ & $0.1<\mathrm{R} 1<1.0$ \\
\hline $\mathrm{R} 2$ & $\mathrm{C}_{2} \mathrm{H}_{2} / \mathrm{C}_{2} \mathrm{H}_{4}$ & $\mathrm{R} 2<0.1$ & $\mathrm{R} 2<0.1$ & $\mathrm{R} 2<0.1$ & $\mathrm{R} 2<0.1$ & $1.0<\mathrm{R} 2<3.0$ \\
\hline R5 & $\mathrm{C}_{2} \mathrm{H}_{4} / \mathrm{C}_{2} \mathrm{H}_{6}$ & $1.0<\mathrm{R} 5<3.0$ & $1.0<\mathrm{R} 5<3.0$ & $3.0<\mathrm{R} 5$ & $\mathrm{R} 5<1.0$ & $3.0<\mathrm{R} 5$ \\
\hline
\end{tabular}

Table 3. The Linear SVM discriminant formula and the a - g coefficient [2].

\begin{tabular}{|c|c|c|c|c|c|c|c|c|c|}
\hline & \multicolumn{9}{|c|}{$\mathrm{Z}=a * \mathrm{H}_{2}+b * \mathrm{CH}_{4}+c * \mathrm{C}_{2} \mathrm{H}_{6}+d * \mathrm{C}_{2} \mathrm{H}_{4}+e * \mathrm{C}_{2} \mathrm{H}_{2}+f * \mathrm{CO}+g$} \\
\hline \multirow{7}{*}{ Coefficient } & Formula & (A) $\mathrm{H}_{2}$ & (B) $\mathrm{CH}_{4}$ & (C) $\mathrm{C}_{2} \mathrm{H}_{6}$ & (D) $\mathrm{C}_{2} \mathrm{H}_{4}$ & (E) $\mathrm{C}_{2} \mathrm{H}_{2}$ & (F) $\mathrm{CO}$ & G & $\begin{array}{c}\text { Diagnosis } \\
\text { result }\end{array}$ \\
\hline & (3) & 0.01815 & -0.01365 & 0.02362 & -0.12971 & -7.32744 & 0.01223 & 2.21713 & Abnormal \\
\hline & $(4)$ & - & - & - & -0.06095 & -11.21398 & - & 5.82626 & Abnormal \\
\hline & (5) & 0.015052 & 0.032666 & -0.019081 & -0.034072 & 0.084326 & -0.002029 & 2.4662 & Overheating \\
\hline & (6) & 0.006088 & -0.046683 & 0.124659 & 0.015673 & -0.06937 & 0.016078 & -1.19747 & Electric Arc \\
\hline & (7) & -0.010295 & -0.068228 & 0.023078 & 0.057307 & 0.16239 & 0.002373 & 0.79497 & $\begin{array}{l}\text { Overheating + } \\
\text { Discharge }\end{array}$ \\
\hline & (8) & -0.033417 & 0.11355 & -0.108216 & 0.029086 & 0.034658 & -0.019222 & 1.1711 & Oil Mixes in \\
\hline
\end{tabular}




\subsection{The Key Gas Method}

When Transformer fault occurred from the gas content of TCG that ones were distinguished into four typical fault types, namely such as Overheating, Corona, Arcing, and Paper Fiber Overheating and so on: 1) Overheating because the insulating oil or insulating paper aging deterioration occurs, then it was decomposed dominating of $\mathrm{C}_{2} \mathrm{H}_{4}(63 \%), \mathrm{C}_{2} \mathrm{H}_{6}(19 \%), \mathrm{CH}_{4}(17 \%)$, and $\mathrm{C}_{2} \mathrm{H}_{2}$ (1\%); 2) Corona was decomposed mainly $\mathrm{H}_{2}(84 \%), \mathrm{CH}_{4}$ (12\%), $\mathrm{C}_{2} \mathrm{H}_{6}$ (3\%), and $\mathrm{C}_{2} \mathrm{H}_{2}(1 \%)$; 3) Arcing was decomposed mainly $\mathrm{H}_{2}(62 \%), \mathrm{C}_{2} \mathrm{H}_{2}(32 \%), \mathrm{CH}_{4}(3 \%), \mathrm{C}_{2} \mathrm{H}_{4}$ (2\%), and $\mathrm{C}_{2} \mathrm{H}_{6}(1 \%)$; 4) Paper Fiber Overheating was decomposed mainly $\mathrm{CO}(92 \%), \mathrm{CH}_{4}(3 \%), \mathrm{H}_{2} \& \mathrm{C}_{2} \mathrm{H}_{6}$ (2\%), and $\mathrm{C}_{2} \mathrm{H}_{4}(1 \%)$. Those gases made up of the rate percentage of each gas that they are shown in Figure 1. By construction of rate of insulating oil composed to diagnosis transformer fault types that were called "the Key Gas method" [5].

\section{The Diagnosis Approach for Practice}

\section{The Design Flow Chart (Show in Figure 2)}

In order to get my diagnostic experience across the field of diagnosis of transformer insulating oil, I made a heuristics program which consists of the ANSI/IEEE C57.104 Standard Rule, the Linear SVM Method, the Roger \& Doernenber Ratios, and the Key Gas Method for diagnosis of transformer incipient fault. Form the flow chart, the transformer's insulating oil through the decomposition chromatographic analysis (ASTM D3612) to produce nine kinds of gases such as $\mathrm{H}_{2}, \mathrm{CH}_{4}, \mathrm{C}_{2} \mathrm{H}_{6}, \mathrm{C}_{2} \mathrm{H}_{4}, \mathrm{C}_{2} \mathrm{H}_{2}, \mathrm{CO}, \mathrm{CO}_{2}, \mathrm{~N}_{2}$, and $\mathrm{O}_{2}$ and so on., While we only took $\mathrm{H}_{2}$, $\mathrm{CH}_{4}, \mathrm{C}_{2} \mathrm{H}_{6}, \mathrm{C}_{2} \mathrm{H}_{4}, \mathrm{C}_{2} \mathrm{H}_{2}$, and $\mathrm{CO}$ for the detective data keyed in the application of program which will be carry out the result of diagnosis by tests. It was shown Figure 2.

\section{Practical Diagnosis and Verification}

\subsection{Diagnostic Practice Case $1 \&$ Case 2}

For provability, from the Siemens Company reported data by Ivanka Atanasova-Hohiein in November of 2014,
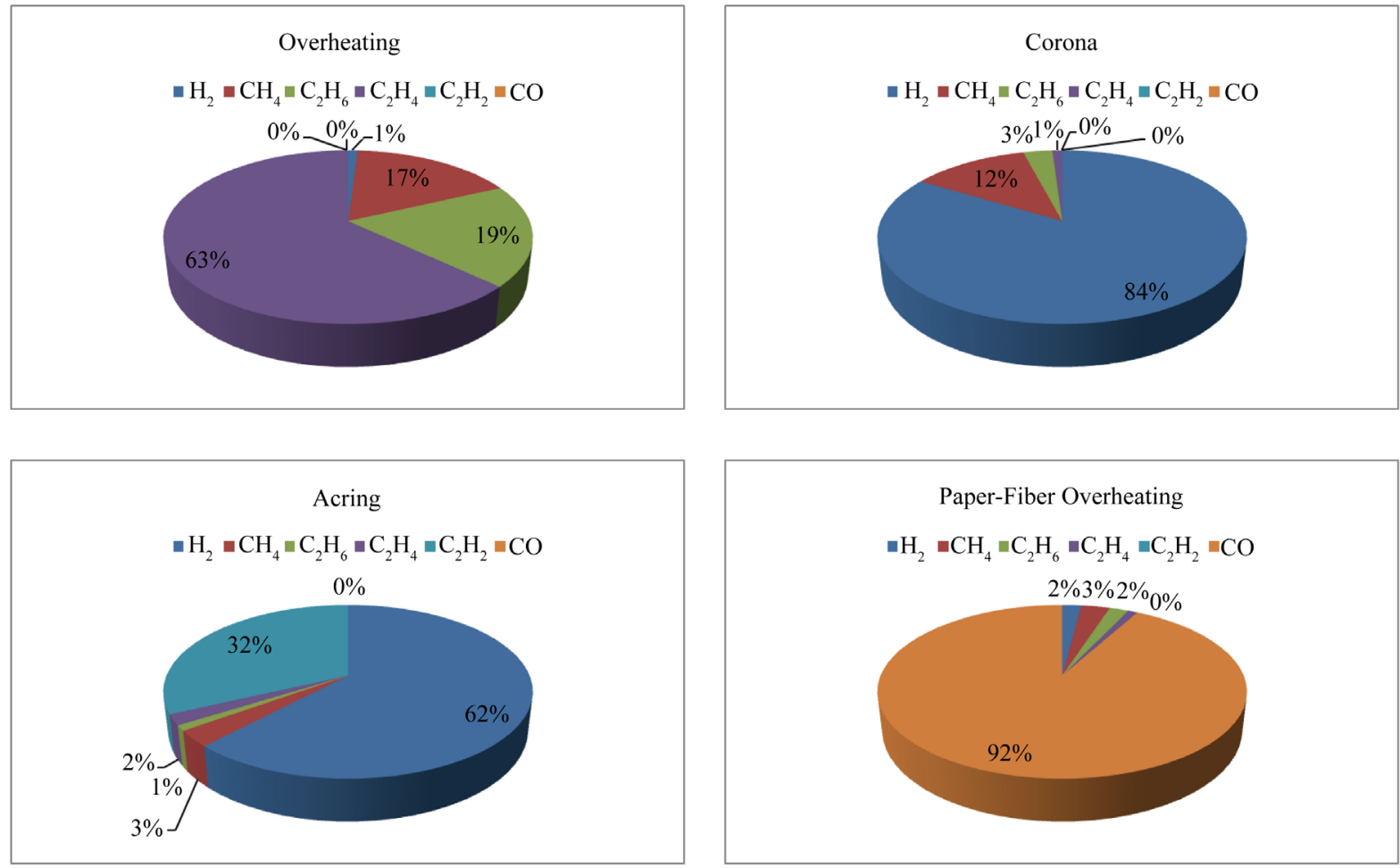

Figure 1. The gas content of typical faults proportion. 


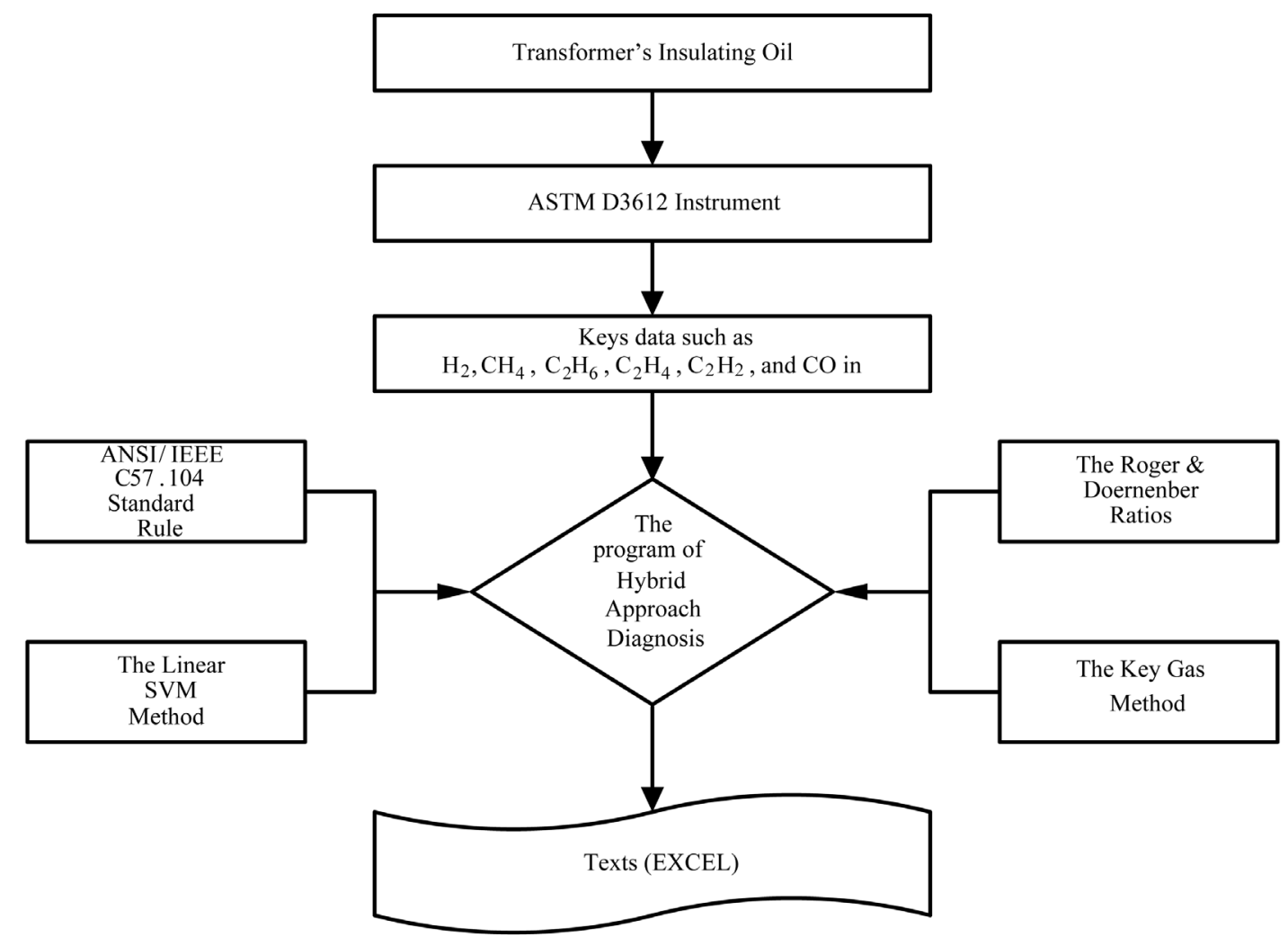

Figure 2. Diagnostic flowchart.

taking two cases data that shown in Table 4 to double check the program, we keyed those number of gases in the diagnostic program then the result of diagnosis that were shown four kinds results in Figure 3 by texts simultaneously [6]. For convenient comparison, taking practically fault pictures of abnormality of phenomenon were shown in Figure 4 and Figure 5 that they were found out from implementing of transformer internal maintenance. From those data verify “A Hybrid Diagnosis Approach of EXCEL program” does provide a quick diagnosis of transformer incipient fault exactly.

\subsection{Take Some Cases to Confirm}

For approving the hybrid diagnosis approach is more validated than others diagnosis ones. Taking four cases in Table 5, exception the case \#4 was validated no incipient fault in others three cases (\#1 - \#3) were confirmed what had incipient fault by transformer's internal maintenance [8]. Then those cases of text description were shown in Table 6 and the programs were shown from case 1 - case 4 in Figure 6. The data of diagnosis were taken from Research Institute of Taiwan Power Company to verify the diagnostic approach that it was well in diagnosis of transformer insulating oil.

\section{Conclusion}

Although it is difficult to be diagnosis and analysis of transformer's insulating oil correctly. Because the amount of gas changes a little that will influence the result of diagnosis a huge, so how to judge that is a significant task. Figures 3-5 data were taken from the report of Siemens Company by Ivanka Atanasova-Hohiein in November of 2014, then Table 6 data, taken from Research Institute of Taiwan Power Company. The data of Figure 3 and Table 5 were taken from known fault transformer insulating oil. The fault point of the figure (Figure 4, Figure 5), that were taken from maintenance in. The purpose of the above is to validate the feasibility of the Hybrid Diagnosis Approach. This paper, the major advantage was not only showed various results of diagnosis out on a report form, but also provided those results to compare analysis. The Hybrid diagnosis approach is validated and confirmed so easy and analysis to use at any condition of transformer insulating oil. This diagnosis approach 
Table 4. Ivanka Atanasova-Hohiein reports gas data in November of 2014 [7], unit: ppm.

\begin{tabular}{cccccccc}
\hline Date & $\mathrm{H}_{2}$ & $\mathrm{CH}_{4}$ & $\mathrm{C}_{2} \mathrm{H}_{6}$ & $\mathrm{C}_{2} \mathrm{H}_{4}$ & $\mathrm{C}_{2} \mathrm{H}_{2}$ & $\mathrm{CO}$ \\
\hline $2012.05(\mathrm{~A})$ & 1967 & 8008 & 2013 & 8323 & 57 & 253 \\
$2012.05(B)$ & 128 & 25 & 5 & 81 & 288 & 143 \\
\hline
\end{tabular}

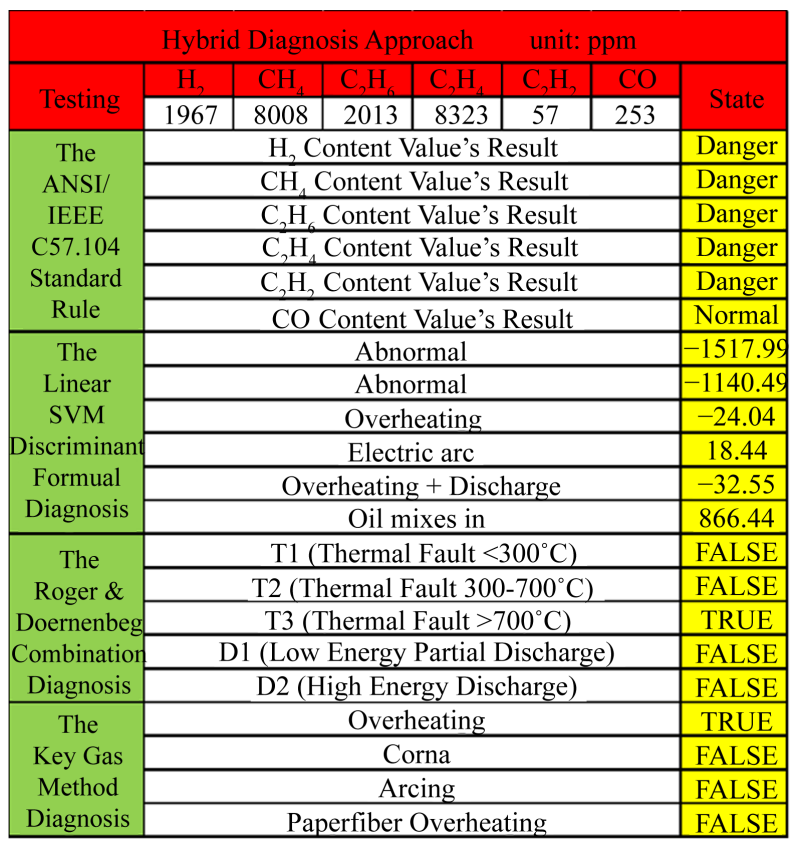

\begin{tabular}{|c|c|c|c|c|c|c|c|}
\hline \multirow{3}{*}{ Testing } & \multicolumn{4}{|c|}{ Hybrid Diagnosis Approach } & unit: & & \multirow{3}{*}{ State } \\
\hline & $\mathrm{H}_{2}$ & $\mathrm{CH}_{\mathrm{H}}$ & $\mathrm{C}_{2} \mathrm{H}_{6}$ & $\mathrm{C}_{2} \mathrm{H}_{4}$ & $\mathrm{C}_{2} \mathrm{H}_{2}$ & $\mathrm{CO}$ & \\
\hline & 128 & 25 & 5 & 81 & 288 & 143 & \\
\hline \multirow{6}{*}{$\begin{array}{l}\text { The } \\
\text { ANSI/ } \\
\text { IEEE } \\
\text { C57.104 } \\
\text { Standard } \\
\text { Rule }\end{array}$} & \multicolumn{6}{|c|}{$\mathrm{H}_{2}$ Content Value's Result } & Attention \\
\hline & \multicolumn{6}{|c|}{$\mathrm{CH}_{4}$ Content Value's Result } & Normal \\
\hline & \multicolumn{6}{|c|}{$\mathrm{C}_{2} \mathrm{H}_{6}$ Content Value's Result } & Normal \\
\hline & \multicolumn{6}{|c|}{$\mathrm{C}_{2} \mathrm{H}_{4}$ Content Value's Result } & Attention \\
\hline & \multicolumn{6}{|c|}{$\mathrm{C}_{2} \mathrm{H}_{2}$ Content Value's Result } & Danger \\
\hline & \multicolumn{6}{|c|}{ CO Content Value's Result } & Normal \\
\hline \multirow{6}{*}{$\begin{array}{c}\text { The } \\
\text { Linear } \\
\text { SVM } \\
\text { Discriminan } \\
\text { Formual } \\
\text { Diagnosis } \\
\end{array}$} & \multicolumn{6}{|c|}{ Abnormal } & -2114.74 \\
\hline & \multicolumn{6}{|c|}{ Abnormal } & -3228.74 \\
\hline & \multirow{2}{*}{\multicolumn{6}{|c|}{ Overheating }} & 26.35 \\
\hline & \multicolumn{2}{|c|}{ Electric arc } & & & & & -17.37 \\
\hline & \multirow{2}{*}{\multicolumn{6}{|c|}{$\frac{\text { Overheating + Discharge }}{\text { Oil mixes in }}$}} & 49.64 \\
\hline & & & & & & & 8.78 \\
\hline \multirow{3}{*}{$\begin{array}{c}\text { The } \\
\text { Roger \& } \\
\text { Doernenbeg }\end{array}$} & \multicolumn{6}{|c|}{$\mathrm{T} 1$ (Thermal Fault $<300^{\circ} \mathrm{C}$ ) } & FALSE \\
\hline & \multicolumn{6}{|c|}{ T2 (Thermal Fault $300-700^{\circ} \mathrm{C}$ ) } & FALSF \\
\hline & \multicolumn{6}{|c|}{ T3 $\left(\right.$ Thermal Fault $\left.>700^{\circ} \mathrm{C}\right)$} & FALSE \\
\hline \multirow{2}{*}{$\begin{array}{c}\text { Combination } \\
\text { Diagnosis } \\
\end{array}$} & \multicolumn{6}{|c|}{ D1 (Low Energy Partial Discharge) } & FALSE \\
\hline & \multicolumn{6}{|c|}{ D2 (High Energy Discharge) } & TRUE \\
\hline \multirow{4}{*}{$\begin{array}{c}\text { The } \\
\text { Key Gas } \\
\text { Method } \\
\text { Diagnosis }\end{array}$} & \multicolumn{6}{|c|}{ Overheating } & FALSE \\
\hline & \multicolumn{6}{|c|}{ Corna } & TRUE \\
\hline & \multirow{2}{*}{\multicolumn{6}{|c|}{ Arcing }} & FALSE \\
\hline & \multicolumn{5}{|c|}{ Paperfiber Overheating } & & FALSE \\
\hline
\end{tabular}

2012.05 (A Case)

2012.05 (B Case)

Figure 3. Transformer fault diagnosis approach.

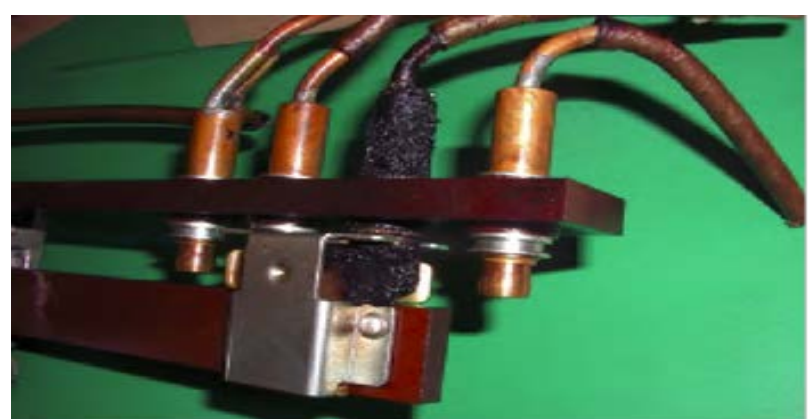

Figure 4. A thermal problem [7].

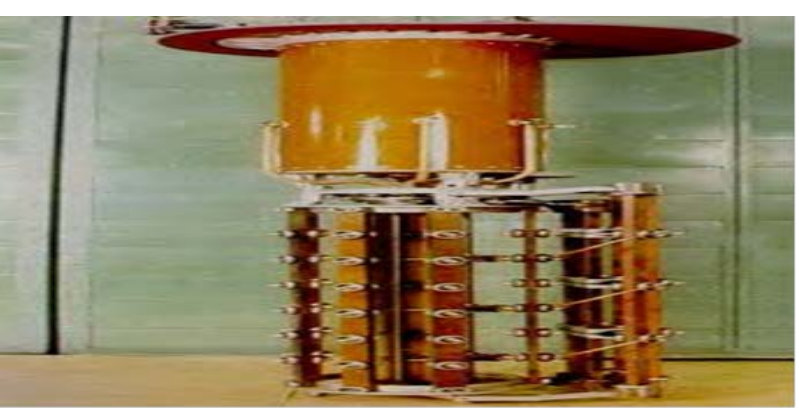

Figure 5. Uptight OLTC [7]. 
Table 5. Some in practical transformer gas data [8], unit: ppm.

\begin{tabular}{ccccccc}
\hline Date & $\mathrm{H}_{2}$ & $\mathrm{CH}_{4}$ & $\mathrm{C}_{2} \mathrm{H}_{6}$ & $\mathrm{C}_{2} \mathrm{H}_{4}$ & $\mathrm{C}_{2} \mathrm{H}_{2}$ & $\mathrm{CO}$ \\
\hline $\mathrm{C} 1(2012.05 .10)$ & 239 & 346 & 78 & 787 & 24 & 312 \\
$\mathrm{C} 2(2012.10 .01)$ & 185 & 601 & 208 & 590 & 1.7 & 72 \\
$\mathrm{C} 3(2013.09 .23)$ & 133 & 211 & 66 & 384 & 1.9 & 411 \\
$\mathrm{C} 4(2012.12 .12)$ & 13 & 3 & 12 & 10 & 0.2 & 310 \\
\hline
\end{tabular}

Table 6. In practical implementation of the results.

\begin{tabular}{|c|c|c|c|c|c|c|c|c|c|c|c|}
\hline \multirow{3}{*}{ Testing } & \multicolumn{3}{|c|}{ Hybrid Diagnosis Approach } & \multicolumn{3}{|c|}{ unit: ppm } & \multirow{3}{*}{ State } & \multirow{3}{*}{$\begin{array}{c}\mathrm{C} 1 \\
\text { State }\end{array}$} & \multirow{3}{*}{$\begin{array}{c}\mathrm{C} 2 \\
\text { State }\end{array}$} & \multirow{3}{*}{$\begin{array}{c}\mathrm{C} 3 \\
\text { State }\end{array}$} & \multirow{3}{*}{$\begin{array}{c}\text { C4 } \\
\text { State }\end{array}$} \\
\hline & $\mathrm{H}_{2}$ & $\mathrm{CH}_{4}$ & $\mathrm{C}_{2} \mathrm{H}_{6}$ & $\mathrm{C}_{2} \mathrm{H}_{4}$ & $\mathrm{C}_{2} \mathrm{H}_{2}$ & $\mathrm{CO}$ & & & & & \\
\hline & 128 & 25 & 5 & 81 & 288 & 143 & & & & & \\
\hline \multirow{2}{*}{ The } & \multicolumn{6}{|c|}{$\mathrm{H}_{2}$ Content Value's Result } & At & At & At & At & $\mathrm{N}$ \\
\hline & \multicolumn{6}{|c|}{$\mathrm{CH}_{4}$ Content Value's Result } & $\mathrm{N}$ & At & $\mathrm{Ab}$ & At & $\mathrm{N}$ \\
\hline ANSI/IEEE & \multicolumn{6}{|c|}{$\mathrm{C}_{2} \mathrm{H}_{6}$ Content Value's Result } & $\mathrm{N}$ & At & $\mathrm{D}$ & At & $\mathrm{N}$ \\
\hline C57.104 & \multicolumn{6}{|c|}{$\mathrm{C}_{2} \mathrm{H}_{4}$ Content Value's Result } & At & $\mathrm{D}$ & $\mathrm{D}$ & $\mathrm{D}$ & $\mathrm{N}$ \\
\hline Standard & \multicolumn{6}{|c|}{$\mathrm{C}_{2} \mathrm{H}_{2}$ Content Value's Result } & $\mathrm{D}$ & $\mathrm{Ab}$ & $\mathrm{N}$ & $\mathrm{N}$ & $\mathrm{N}$ \\
\hline \multirow[t]{2}{*}{ Rule } & \multicolumn{6}{|c|}{ CO Content Value's Result } & $\mathrm{N}$ & $\mathrm{N}$ & $\mathrm{N}$ & At & $\mathrm{N}$ \\
\hline & \multicolumn{6}{|c|}{ Abnormal } & -2114 & -270 & -85 & -55 & 3 \\
\hline The Linear & \multicolumn{6}{|c|}{ Abnormal } & -3228 & -311 & -49 & -38 & 2 \\
\hline SVM & \multicolumn{6}{|c|}{ Overheating } & 26 & -9 & 0 & -3 & 1 \\
\hline Discriminant & \multicolumn{6}{|c|}{ Electric arc } & -17 & 9 & 8 & 10 & 5 \\
\hline Formual & \multicolumn{6}{|c|}{ Overheating + Discharge } & 49 & 26 & -3 & 9 & 2 \\
\hline \multirow[t]{2}{*}{ Diagnosis } & \multicolumn{6}{|c|}{ Oil mixes in } & 8 & 41 & 56 & 16 & -5 \\
\hline & \multicolumn{6}{|c|}{$\mathrm{T} 1$ (Thermal Fault $<300^{\circ} \mathrm{C}$ ) } & False & False & True & False & False \\
\hline The Roger \& & & $\mathrm{T} 2(\mathrm{Th}$ & nal Fault & $00^{\circ} \mathrm{C}-$ & $\left.10^{\circ} \mathrm{C}\right)$ & & False & False & False & False & False \\
\hline Doernenbeg & & $\mathrm{T} 3$ & ermal F & lt $>700$ & & & False & True & False & True & False \\
\hline Combination & & D1 (Lo & Energy $\mathrm{P}$ & tial Dis & large) & & False & False & False & False & False \\
\hline Diagnosis & & $\mathrm{D} 2$ & gh Energ & Discha & & & True & False & False & False & False \\
\hline & & & Overhe & ing & & & False & True & True & True & False \\
\hline The Key Gas & & & Cor & & & & True & False & False & False & False \\
\hline Method & & & Arci & & & & False & False & False & False & False \\
\hline Diagnosis & & & $\mathrm{r}$-fiber $\mathrm{C}$ & erheatir & & & False & False & False & False & False \\
\hline
\end{tabular}

Symbols: N (Normal), Ab (Abnormal), At (Attention), D (Danger), F (False). 


\begin{tabular}{|c|c|c|c|c|c|c|c|}
\hline & \multicolumn{4}{|c|}{ Hybrid Diagnosis Approach } & \multicolumn{2}{|c|}{ unit: ppm } & \multirow{3}{*}{ State } \\
\hline \multirow{2}{*}{ Testing } & $\mathrm{H}_{2}$ & $\mathrm{CH}_{4}$ & $\mathrm{C}_{2} \mathrm{H}_{6}$ & $\mathrm{C}_{2} \mathrm{H}_{4}$ & $\mathrm{C}_{2} \mathrm{H}_{2}$ & $\mathrm{CO}$ & \\
\hline & 239 & 346 & 78 & 787 & 24 & 312 & \\
\hline \multirow{6}{*}{$\begin{array}{l}\text { The } \\
\text { ANSI/ } \\
\text { IEEE } \\
\text { C57.104 } \\
\text { Standard } \\
\text { Rule }\end{array}$} & \multicolumn{6}{|c|}{$\mathrm{H}_{2}$ Content Value's Result } & Attention \\
\hline & \multicolumn{6}{|c|}{$\mathrm{CH}_{4}$ Content Value's Result } & Attention \\
\hline & \multicolumn{6}{|c|}{$\mathrm{C}_{2} \mathrm{H}_{6}$ Content Value's Result } & Attention \\
\hline & \multicolumn{6}{|c|}{$\mathrm{C}_{2} \mathrm{H}_{4}$ Content Value's Result } & Danger \\
\hline & \multicolumn{6}{|c|}{$\mathrm{C}_{2} \mathrm{H}_{2}$ Content Value's Result } & Abnorma \\
\hline & \multicolumn{6}{|c|}{ CO Content Value's Result } & Normal \\
\hline \multirow{6}{*}{$\begin{array}{c}\text { The } \\
\text { Linear } \\
\text { SVM } \\
\text { Discriminan } \\
\text { Formual } \\
\text { Diagnosis }\end{array}$} & \multicolumn{6}{|c|}{ Abnormal } & -270.45 \\
\hline & \multicolumn{6}{|c|}{ Abnormal } & -311.26 \\
\hline & \multicolumn{6}{|c|}{ Overheating } & -9.55 \\
\hline & \multicolumn{6}{|c|}{ Electric arc } & -9.51 \\
\hline & \multicolumn{6}{|c|}{ Overheating + Discharge } & 26.27 \\
\hline & \multicolumn{6}{|c|}{ Oil mixes in } & 41.76 \\
\hline \multirow{5}{*}{$\begin{array}{c}\text { The } \\
\text { Roger \& } \\
\text { Doernenbeg } \\
\text { Combination } \\
\text { Diagnosis }\end{array}$} & \multicolumn{6}{|c|}{$\mathrm{T} 1$ (Thermal Fault $<300^{\circ} \mathrm{C}$ ) } & FALSE \\
\hline & \multicolumn{6}{|c|}{$\mathrm{T} 2$ (Thermal Fault $300-700^{\circ} \mathrm{C}$ ) } & FALSE \\
\hline & \multicolumn{6}{|c|}{$\mathrm{T} 3\left(\right.$ Thermal Fault $\left.>700^{\circ} \mathrm{C}\right)$} & TRUE \\
\hline & \multicolumn{6}{|c|}{ D1 (Low Energy Partial Discharge) } & FALSE \\
\hline & \multicolumn{6}{|c|}{ D2 (High Energy Discharge) } & FALSE \\
\hline \multirow{4}{*}{$\begin{array}{l}\text { The } \\
\text { Key Gas } \\
\text { Method } \\
\text { Diagnosis }\end{array}$} & \multicolumn{6}{|c|}{ Overheating } & TRUE \\
\hline & \multicolumn{6}{|c|}{ Corna } & FALSE \\
\hline & \multicolumn{6}{|c|}{ Arcing } & FALSE \\
\hline & \multicolumn{6}{|c|}{ Paperfiber Overheating } & FALSE \\
\hline
\end{tabular}

2012.05.10 (Case 1)

\begin{tabular}{|c|c|c|c|c|c|c|c|}
\hline & \multicolumn{4}{|c|}{ Hybrid Diagnosis Approach } & \multicolumn{2}{|c|}{ unit: ppm } & \multirow{3}{*}{ State } \\
\hline \multirow{2}{*}{ Testing } & $\mathrm{H}_{2}$ & $\mathrm{CH}_{4}$ & $\mathrm{C}_{2} \mathrm{H}_{6}$ & $\mathrm{C}_{2} \mathrm{H}_{4}$ & $\mathrm{C}_{2} \mathrm{H}_{2}$ & $\mathrm{CO}$ & \\
\hline & 133 & 211 & 66 & 384 & 1.9 & 411 & \\
\hline \multirow{6}{*}{$\begin{array}{l}\text { The } \\
\text { ANSI/ } \\
\text { IEEE } \\
\text { C57.104 } \\
\text { Standard } \\
\text { Rule }\end{array}$} & \multicolumn{6}{|c|}{$\mathrm{H}_{2}$ Content Value's Result } & Attention \\
\hline & \multicolumn{6}{|c|}{$\mathrm{CH}_{4}$ Content Value's Result } & Attention \\
\hline & \multicolumn{6}{|c|}{$\mathrm{C}_{2} \mathrm{H}_{6}$ Content Value's Result } & Attention \\
\hline & \multicolumn{6}{|c|}{$\mathrm{C}_{2} \mathrm{H}_{4}$ Content Value's Result } & Danger \\
\hline & \multicolumn{6}{|c|}{$\mathrm{C}_{2} \mathrm{H}_{2}$ Content Value's Result } & Normal \\
\hline & \multicolumn{6}{|c|}{ CO Content Value's Result } & Attention \\
\hline \multirow{6}{*}{$\begin{array}{c}\text { The } \\
\text { Linear } \\
\text { SVM } \\
\text { Discriminan } \\
\text { Formual } \\
\text { Diagnosis }\end{array}$} & \multicolumn{6}{|c|}{ Abnormal } & -55.39 \\
\hline & \multicolumn{6}{|c|}{ Abnormal } & -38.88 \\
\hline & \multicolumn{6}{|c|}{ Overheating } & -3.66 \\
\hline & \multicolumn{6}{|c|}{ Electric arc } & 10.48 \\
\hline & \multicolumn{6}{|c|}{ Overheating + Discharge } & 9.84 \\
\hline & \multicolumn{6}{|c|}{ Oil mixes in } & 16.88 \\
\hline \multirow{5}{*}{$\begin{array}{c}\text { The } \\
\text { Roger \& } \\
\text { Doernenbeg } \\
\text { Combination } \\
\text { Diagnosis }\end{array}$} & \multicolumn{6}{|c|}{ T1 (Thermal Fault $<300^{\circ} \mathrm{C}$ ) } & FALSE \\
\hline & \multicolumn{6}{|c|}{ T2 (Thermal Fault $300-700^{\circ} \mathrm{C}$ ) } & FALSE \\
\hline & \multicolumn{6}{|c|}{$\mathrm{T} 3$ (Thermal Fault $\left.>700^{\circ} \mathrm{C}\right)$} & TRUE \\
\hline & \multicolumn{6}{|c|}{ D1 (Low Energy Partial Discharge) } & FALSE \\
\hline & \multicolumn{6}{|c|}{ D2 (High Energy Discharge) } & FALSE \\
\hline \multirow{4}{*}{$\begin{array}{c}\text { The } \\
\text { Key Gas } \\
\text { Method } \\
\text { Diagnosis }\end{array}$} & \multicolumn{6}{|c|}{ Overheating } & TRUE \\
\hline & \multicolumn{6}{|c|}{ Corna } & FALSE \\
\hline & \multicolumn{6}{|c|}{ Arcing } & FALSE \\
\hline & \multicolumn{6}{|c|}{ Paperfiber Overheating } & FALSE \\
\hline
\end{tabular}

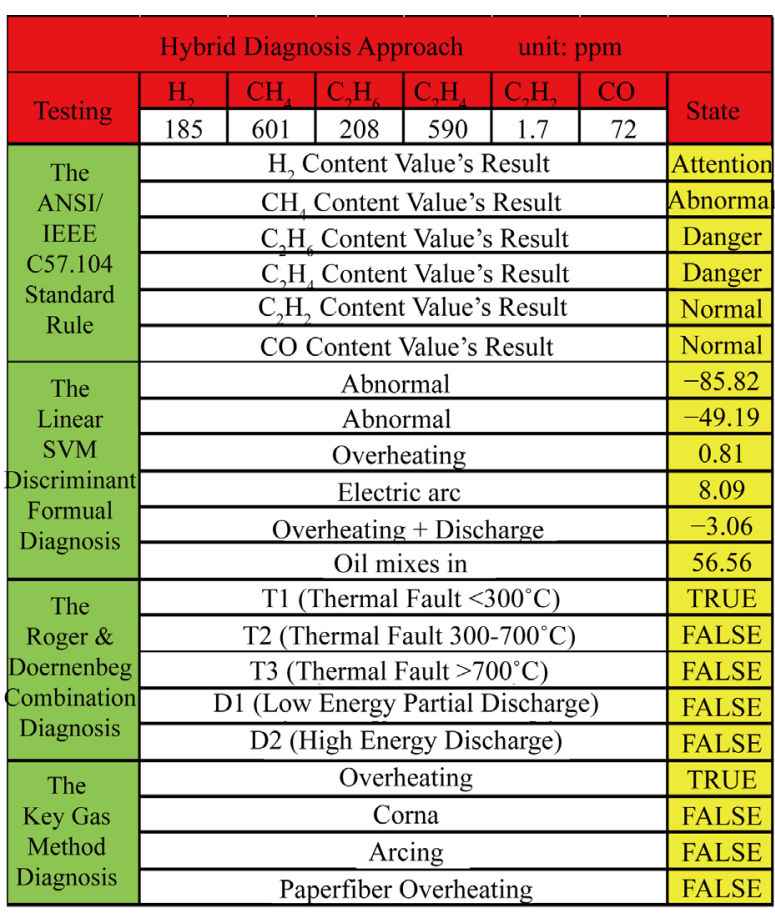

2012.10.01 (Case 2)

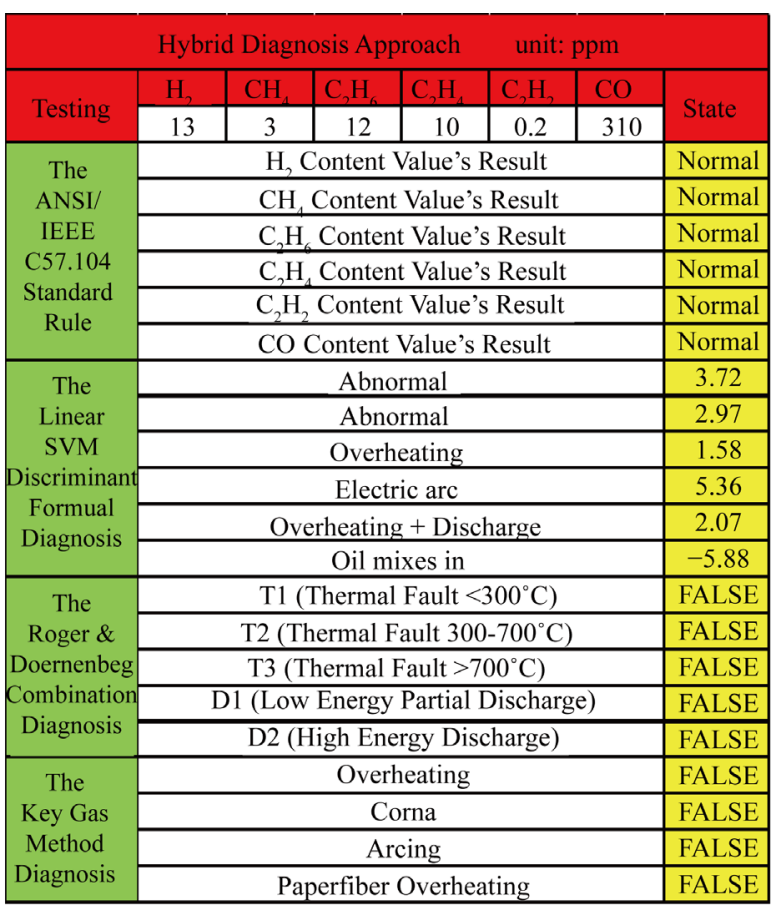

2012.12.12 (Case 4)

Figure 6. Transformer fault diagnosis approach for C1 - C4.

will be useful for engineers and technicians those who are in charge of transformer's maintenance.

\section{References}

[1] IEEE (2012) C57.104_IEEE Guide for the Interpretation of Gases Generated in Oil—Immersed Transformers. Minutes of WG Meeting Nashville, Tennessee. 
[2] Mollman, A. and Pahlavanpour, B. (1999) New Guidelines for Interpretation of Dissolved Gas Analysis in Oil-Filled Transformers. Electra, CIGRE France, 186, 30-51.

[3] IEC (1999) Guide to the Interpretation of Dissolved and Free Gasses Analysis. IEC Standard 60599, IEC Publ. 60599.

[4] Singh, S. and Bandyopadhyay, M.N. (2010) Duval Triangle: A Noble Technique for DGA in Power Transformers. International Journal of Electrical and Power Engineering, 4, 193-197. http://dx.doi.org/10.3923/ijepe.2010.193.197

[5] Electric Cooperative Research Council (2009) The Revised Guidelines of Power Transformers. Vol. 1, Heisei 21 Years.

[6] Qi, Y.G., Ming, L.K., Sing, G.B., Sung, S.U. and Xia, S. (2012) Nan Cou E/S \#4 ATr Overhaul Report. Taiwan Power Company Institute.

[7] Atanasova-Höhlein, I. (2014) DGA-Method in the Past and for the Future. Siemens Company. https://www.energy.siemens.com/mx/pool/hq/services/power-transmission

[8] Than, C.F., Qing, C.J. and Than, L.Y. (2012) Nan Cou E/S \# 4 ATr Overhaul Report. Future Electric Co. Ltd. 